\title{
Social status and the quality of care for adult people with Type I (insulin-dependent) diabetes mellitus - a population-based study
}

\author{
I. Mühlhauser ${ }^{1}$, H. Overmann ${ }^{1}$, R. Bender ${ }^{1}$, U. Bott ${ }^{1}$, V.Jörgens ${ }^{1}$, Ch. Trautner ${ }^{1}$ J. Siegrist $^{2}$, M. Berger ${ }^{1}$ \\ ${ }^{1}$ Department of Metabolic Diseases and Nutrition (WHO-Collaborating Centre for Diabetes), Heinrich-Heine University, \\ Düsseldorf, Germany \\ ${ }^{2}$ Institute of Medical Sociology, Heinrich-Heine University, Düsseldorf, Germany
}

Summary The objective of this study was to assess the degree of diabetes care and education achieved for Type I (insulin-dependent) diabetes mellitus at the community level in relation to social status and to elucidate potential pathways that mediate any social class gradient. A population-based sample of 684 adults with Type I diabetes ( $41 \%$ women, mean \pm SD age $36 \pm 11$, diabetes duration $18 \pm$ 11 years) in the district of North-Rhine (9.5 million inhabitants), Germany, were examined in their homes using a mobile ambulance. Results: $\mathrm{HbA}_{1 \mathrm{c}}$ (normal $4.3-6.1 \%$ ) $8.0 \pm 1.5 \%$, incidence of severe hypoglycaemia (injection of glucose or glucagon) 0.21 cases per patient-year; $62 \%$ of patients had participated in a structured group treatment and teaching programme for intensification of insulin therapy; $70 \%$ used 3 or more insulin injections per day, $9 \%$ were on continuous subcutaneous insulin infusion; $91 \%$ reported to have had measurements of $\mathrm{HbA}_{1 \mathrm{c}}$ during the preceding year, and $80 \%$ to have had an examination of the retina by an ophthalmologist. Care was insufficient with respect to the quality of blood pressure control $(70 \%$ of patients on antihypertensive drugs had blood pressure values $\geq 160$ / $95 \mathrm{mmHg}$ ), patient awareness of proteinuria/albuminuria $(27 \%$ of patients had not heard about it) and prevention of foot complications (only $42 \%$ with a diabetes duration over 10 years had remembered to have a foot examination during the preceding 12 months). There was a pronounced social gradient with respect to micro- and macrovascular complications (prevalence of overt nephropathy 7 vs $20 \%$ for highest vs lowest quintiles of social class [OR 3.5, $95 \%$ CI 1.6-7.5, $p=0.002]$ ) and diabetes-specific quality of life. $\mathrm{HbA}_{1 \mathrm{c}}$, blood pressure and smoking accounted for part of the association between social class and microvascular complications. The social class gradient was not due to inequality to access to health services, but to lower acceptance among low social class patients of preventive and health maintaining behaviour. In conclusion, achieved standards of care are high with respect to the implementation of intensified treatment regimens, the level of patient education achieved, treatment control and eye care, whereas areas for improvement are blood pressure control and preventive measures for foot care. A substantial social gradient in diabetes care persists despite equal access of patients to health services. [Diabetologia (1998) 41: 1139-1150]

Keywords Type I (insulin-dependent) diabetes, quality of care, diabetes education, late complications, $\mathrm{HbA}_{1 \mathrm{c}}$, hypoglycaemia, diet, cardiovascular complications, quality of life.
Received: 29 September 1997 and in revised form: 8 April 1998

Corresponding author: Univ.-Prof. Dr. med. Ingrid Mühlhauser, Medizinische Klinik der Universität Düsseldorf, Klinik für Stoffwechselkrankheiten und Ernährung, Moorenstrasse 5, D-40225 Düsseldorf, Germany

Abbreviations: DTTP, Diabetes treatment and teaching programme; CSII, continuous subcutaneous insulin infusion; OR, odds ratio; CI, confidence interval; PWTSS, preferenceweighted treatment satisfaction score; CAPD, continuous ambulatory peritoneal dialysis.
During recent years substantial changes have taken place in the care of persons with Type I (insulin-dependent) diabetes. The implementation of structured diabetes treatment and teaching programmes (DTTP) for intensification of insulin therapy is regarded as the basis for a reduction of diabetes-related acute and late complications, improvement of diabetes care and saving of health care costs by reductions in hospitalisations and periods of sick leave [1]. However, it has been argued that there might be a selec- 
tion in favour of motivated and better-educated patients to participate in such programmes and that lower social class patients are less likely to profit from these modern treatment facilities.

It is well known that the social class factor is an important predictor of morbidity and mortality in the general population, even in highly industrialised countries [2], although controversy persists about how much of this social class gradient can be explained by known cardiovascular risk factors $[3,4]$.

In tertiary care centre based or small case-control studies of Type I diabetes, lower social class has been found to be associated with a higher rate of treatment complications and to be an independent predictor of mortality [5-7].

There have been no population-based comprehensive assessments of the quality of care of representative groups of patients with Type I diabetes including socioeconomic and quality of life aspects.

The aim of this population-based study was to assess the degree of diabetes care and education achieved at the community level in the district of North-Rhine, Germany. Social status of the patients was particularly considered and elucidation of potential pathways that may mediate any relation between social status and quality of care.

\section{Subjects, materials and methods}

Study hypotheses. The study was based upon the following hypotheses: lower social class is associated with: 1) Higher rates of diabetic late complications (nephropathy, retinopathy). 2) Lower degrees of control of risk factors for diabetic late complications $\left(\mathrm{HbA}_{1 \mathrm{c}}\right.$, blood pressure, smoking). 3) Less intensive disease management by health care providers as reflected by fewer diabetes-related diagnostic tests $\left(\mathrm{HbA}_{1 \mathrm{c}}\right.$, blood pressure, neurologic foot examination), less prescription or recommendation of modern treatment regimens (multiple daily insulin injections, continuous subcutaneous insulin infusion (CSII)), less provision of modern facilities for disease management (insulin injection pen devices, glucose meters), and less referral by health care providers to specialised institutions for diabetes care (inpatient DTTP, diabetes outpatient clinic, ophthalmologist). 4) Lower degree of disease related knowledge (on $\mathrm{HbA}_{1 \mathrm{c}}$, acetonuria testing, proteinuria/albuminuria testing), disease/symptom awareness (of having retinopathy, nephropathy, neurologic foot complications), motivation (treatment goals), appropriate disease management behaviour (blood glucose self-monitoring, adaptation of insulin dosages, participation in a DTTP, consultation of a diabetologist), and health promoting behaviour (not smoking). 5) Lower treatment satisfaction, and diabetes-specific quality of life.

Social status classification. Classification of social status was based upon instruments used in previous health surveys in Germany $[8,9]$. The social class score was an additive variable of the highest educational level achieved (scores $0-8$ ), the present or last employment level (scores 0-8) and household income (scores $0-8$ ) resulting in a score ranging from 0 to 24 , higher scores indicating higher levels of social class. Nett income per household was adjusted for number and age of household members in order to calculate the so-called equivalent income per subject [8]. The mean equivalent income of the total patient group was DM 1780 which is comparable to the equivalent income of DM 1720 for the West German population as calculated for the year 1992 [10]. For descriptive purposes quintiles of the total score were used to divide the patient group into five levels of social class (level I corresponding to the highest and level V to the lowest social class).

Recruitment of physicians and patients. Detailed descriptions of the protocol of this population-based study including selection criteria of physicians and patients, participation, and description of drop outs have have been published [11]. Between November 1994 and July 1996 patients were recruited from a random sample of 630 family physician practices using computer lists of the physicians' chamber of North-Rhine, where all physicians are registered. This corresponds to approximately $10 \%$ of all primary health care physicians of North-Rhine. Due to the German health care system patients are not registered with a primary health care physician. It has been estimated that on average each family physician takes care of one to two adults with Type I diabetes. Physicians were asked to obtain written consent to be sent the study protocol and to be contacted by the research team of the Düsseldorf University from each patient fulfilling the inclusion criteria. Physicians were reimbursed for reporting the number of patients fulfilling the inclusion criteria and for each written consent form. Inclusion criteria for patients were: age 18 years and above, initiation of insulin therapy before age 31 years, at least one visit to the respective practice during the preceding 12 months; nonGerman citizens, mentally disabled and nursing home patients were to be included. In order to ascertain complete registration of all available patients physicians and their paramedical assistants were additionally contacted by phone. A total of 2830 phone calls by I. M. with the practices were documented. Physicians of $30(5 \%)$ practices refused to participate. At the end of recruitment $56(9 \%)$ practices had not yet sent written consent forms from their patients; $173(27 \%)$ practices did not take care of Type I diabetic patients fulfilling the inclusion criteria at the time of the study. The remaining 371 practices reported the care for 932 patients fulfilling the inclusion criteria. Of these patients $58(6 \%)$ did not give their written consent, from a further $119(13 \%)$ patients the written consent was not yet available at the end of the study. Of the remaining 755 patients $684(91 \%)$ participated in the study, whereas 58 $(8 \%)$ had declined to participate after having received the study protocol, 7 had moved outside the study area and 6 could not be contacted until the end of the study. The study protocol was approved by the ethics committee of Düsseldorf University.

Evaluation protocol. The examinations were performed in a mobile ambulance as described previously [12] by two principal investigators (I.M. and H.O.) and, on occasion, by other trained investigators (see acknowledgements). The ambulance was converted to allow the mounting of a non-mydriatic CR4-45NM eye camera (Canon Europe, Amstelveen, The Netherlands), and a small laboratory including a Reflotron Reflectance Photometer (Boehringer Mannheim, Mannheim, Germany). Imobile patients were examined in their homes.

The examination protocol used an extended and adapted version of evaluation protocols used in previous studies and included all items of the St. Vincent Declaration data collection form [12-15]. Evaluation forms are available on request from the authors. The examination took about 90 to $120 \mathrm{~min}$ for each patient and comprised a structured interview, self-administered questionnaires, physical examination, fundus photogra- 
phy, and laboratory tests. The interview included questions assessing participant characteristics, such as sociodemographic and health status information, along with information on diabetes care and self-management across multiple areas, and questions assessing potential social, behavioural, and health care provider related correlates of self-management.

A venous blood sample and a freshly voided urine sample were taken from each patient and transported on ice to the laboratory of Düsseldorf University Hospital. $\mathrm{HbA}_{1 \mathrm{c}}$ was measured by the Diamat HPLC-method (reference range 4.3-6.1\%). Random C-peptide levels were measured using a human C-peptide assay (Behring, Marburg, Germany). A value of less than $0.1 \mathrm{nmol} / \mathrm{l}$ was considered negative. Protein concentration in the urine was measured by the laser turbidimetric method [16]. Body weight was assessed with the patients wearing street clothes and without shoes. Sitting blood pressure was measured twice using a mercury sphygmomanometer according to World Health Organisation (WHO) recommendations including the use of a large cuff for patients with increased upper arm circumferences, and the second blood pressure measurement was used for analysis. Smoking history was assessed as described previously [15]. Cigarette pack years were calculated for each patient, and one cigarette pack-year was equivalent to smoking 20 cigarettes per day for one year. Examination of the feet included palpation of pulses and screening for sensation loss using the Rydel-Seiffer-Tuning Fork as described by Liniger et al. [17]. In the case of a foot lesion or an ulcer, a photograph was taken for documentation. Patients were grouped according to (neuropathic) foot complications: level 1: vibration sensation score at the first metatarsal $>4 / 8$ bilaterally, no ulcer, no amputation; level 2: vibration sensation score $\leq 4 / 8$, no ulcer, no amputation; level 3: acute or healed ulcer or amputation. In addition, patients were grouped according to renal parameters: level 1: proteinuria $\leq 50 \mathrm{mg} / \mathrm{l}$ and serum creatinine $\leq 133 \mu \mathrm{mol} / \mathrm{l}$; level 2 : proteinuria $51-499 \mathrm{mg} / \mathrm{l}$ and serum creatinine $\leq 133 \mu \mathrm{mol} / \mathrm{l}$; level 3: proteinuria $\geq 500 \mathrm{mg} / \mathrm{l}$ and serum creatinine $\leq 133 \mu \mathrm{mol} / \mathrm{l}$; level 4 : serum creatinine $>133 \mu \mathrm{mol} / \mathrm{l}$; level 5: renal replacement therapy.

Non-mydriatic photography was performed in the mobile ambulance. In all subjects a macula-centred photograph was obtained of one eye. If there was any hint of retinopathy on this first photograph, a picture of the other retina was taken as well. In cases where the pupils did not sufficiently dilate, whenever possible, pharmacological mydriasis was induced with $1 \%$ tropicamide eye drops. All photographs were examined by two independent experts in Düsseldorf. In addition, patients were given a Diabetic Retinopathy Examination Chart [18] and asked to consult their board certified ophthalmologists and to send back one copy of the filled-in examination chart to the Düsseldorf Study Center. All available eye examination results were used in order to rate the degree of retinopathy for each patient. In case the degree of retinopathy differed between both eyes, and in case the findings differed between methods, the higher degree of retinopathy was used. Best visual acuity was assessed in the ambulance using the visual acuity charts of Ferris et al. [19]. Patients were grouped according to the degree of retinopathy: level 1: no retinopathy; level 2: non-proliferative retinopathy without macular involvement, no history of laser therapy; level 3: pre-proliferative and proliferative retinopathy, macula involvement, macula oedema, history of laser therapy, advanced diabetic eye disease; level 4: blindness of one or both eyes due to diabetes (defined as legal blindness or best visual acuity $\leq 0.1$ ).

Self-administered instruments were used in order to assess diabetes-specific quality of life including preference-weighted treatment satisfaction. A detailed description of these tests in- cluding validation procedures is published elsewhere [20]. After factor analysis, the diabetes-specific quality of life scale comprised 59 items which were rated by the patients on a 6 point Likert scale: 1 . Treatment goals (10 items); 2 . Treatment satisfaction according to treatment goals (10 items); 3 . Social relations (11 items): "Diabetes again and again leads me to problems with other people." "Because of my diabetes it is much harder to make friends." 4. Leisure time flexibility (6 items): "Diabetes prevents me from spontaneous physical activities." "Because of my disease I can't spend my leisure the way I would like to." 5. Physical complaints (8 items): "I feel dull or sluggish." "Because of diabetes I often have physical troubles." 6. Worries about the future (5 items): "I am often worried about diabetic late complications." "I often ponder over diabetes and its consequences." 7. Diet restrictions (5 items): "It bothers me that I can't eat like other people." "I have to give up tasty food." 8 . Daily hassles (4 items): "It bothers me that I have to spend so much time on my diabetes treatment." "It bothers me that I have to take my medical instruments (e.g. syringes) with me, whatever I do." To estimate a preference-weighted treatment satisfaction score (PWTSS), patients' rating for each treatment goal $(1=$ totally unimportant; 6 = very important) was multiplied by the corresponding degree of satisfaction with the achievment in these treatment goals $(-2.5=$ totally dissatisfied; $2.5=$ very satisfied $)$. The sum of all 10 products provided the PWTSS [20]. Cronbach's alpha for all subscales ranged between 0.70 and 0.88 .

Statistical analyses. For comparison of means analysis of variance and for comparison of proportions Fisher's exact test was used. For calculation of $95 \%$ confidence intervals for odds ratios based upon simple $2 \times 2$ tables the Mantel-Haenszel method was used. In order to investigate the effect of social status on various response variables with adjustment for age and diabetes duration a number of multiple regression models were calculated. We applied linear regression for continuous response, logistic regression for binary response and the proportional odds model for ordinal response. If the assumption of proportional odds was violated the ordinal response was dichotomized and binary logistic regression was applied. Social status, age and diabetes duration were used as continuous covariates. To adjust for age and diabetes duration the linear and quadratic terms were considered. Hence, all models contained the covariate social status together with the appropriate linear and quadratic terms of age and diabetes duration. For the responses retinopathy and nephropathy the additional covariates $\mathrm{HbA}_{1 \mathrm{c}}$, blood pressure and cigarette pack years were considered. The validity of the regression models was checked by scatter plots, residual plots, the Hosmer-Lemeshow goodness-of-fit test and the score test for the proportional odds assumption. To assess the effect size of social status adjusted odds ratios based upon logistic regression were calculated for a difference of 5 units (corresponds to approximately 1 social class level). All hypotheses tests were two-tailed. A $p$-value below 0.05 was regarded as significant. Due to the hypothesesgenerating character of the study no adjustments for multiple hypotheses testing were performed. For computations the statistics packages SPSS and SAS were used.

\section{Results}

Mean age of the total group was 36 (range 18 to 77 ) years and mean diabetes duration was 18 (0.3 to 55) years with some differences across social status 
Table 1. Patient characteristics and metabolic control in relation to social status

\begin{tabular}{|c|c|c|c|c|c|c|c|c|c|c|c|}
\hline $\begin{array}{l}\text { Social } \\
\text { status }\end{array}$ & $n$ & $\begin{array}{l}\text { Gender } \\
\text { (\% } \\
\text { women) }\end{array}$ & $\begin{array}{l}\text { Age } \\
\text { (years) }\end{array}$ & $\begin{array}{l}\text { Diabetes } \\
\text { duration } \\
\text { (years) }\end{array}$ & $\begin{array}{l}\text { Body } \\
\text { mass } \\
\text { index } \\
\left(\mathrm{kg} \cdot \mathrm{m}^{-2}\right)\end{array}$ & $\begin{array}{l}\text { C-peptide } \\
\text { negative } \\
\text { (\% patients) }\end{array}$ & $\begin{array}{l}\mathrm{HbA}_{1 \mathrm{c}} \\
(\%)\end{array}$ & $\begin{array}{l}\text { Ketoacido- } \\
\text { sisa since } \\
\text { diabetes } \\
\text { manifesta- } \\
\text { tion/during } \\
\text { preceding } \\
\text { year } \\
\text { (\% patients) }\end{array}$ & $\begin{array}{l}\text { Mild hypo- } \\
\text { glycaemiaa } \\
\text { during pre- } \\
\text { ceding } \\
\text { week (\% } \\
\text { patients) } \\
\text { ) }\end{array}$ & $\begin{array}{l}\text { Severe hypo- } \\
\text { glycaemiaa } \\
\text { since diabe- } \\
\text { tes manife- } \\
\text { station/dur- } \\
\text { ing preceding } \\
\text { year } \\
\text { (\% patients) }\end{array}$ & $\begin{array}{l}\text { Carrying } \\
\text { emergency } \\
\text { carbohy- }^{\text {arbotes }} \\
\text { drates }^{\mathrm{a}} \\
\text { (\% pati- } \\
\text { ents) }\end{array}$ \\
\hline I & 131 & 42 & $36 \pm 10$ & $19 \pm 11$ & $24.3 \pm 2.8$ & $91(n=125)$ & $7.6 \pm 1.2$ & $14 / 0$ & 72 & $46 / 13$ & 87 \\
\hline II & 141 & 41 & $33 \pm 8$ & $15 \pm 9$ & $24.2 \pm 2.7$ & $89(n=136)$ & $7.9 \pm 1.3$ & $13 / 2$ & 70 & $46 / 13$ & 84 \\
\hline III & 136 & 40 & $36 \pm 10$ & $18 \pm 11$ & $25.0 \pm 4.0$ & $89(n=134)$ & $8.0 \pm 1.5$ & $31 / 2$ & 68 & $50 / 13$ & 89 \\
\hline IV & 146 & 36 & $38 \pm 12$ & $19 \pm 12$ & $24.8 \pm 3.6$ & $86(n=146)$ & $8.2 \pm 1.6$ & $23 / 3$ & 54 & $45 / 12$ & 83 \\
\hline V & 130 & 48 & $39 \pm 12$ & $20 \pm 12$ & $24.9 \pm 3.7$ & $80(n=127)$ & $8.4 \pm 1.7$ & $31 / 5$ & 62 & $51 / 12$ & 83 \\
\hline All & 684 & 41 & $36 \pm 11$ & $18 \pm 11$ & $24.6 \pm 3.4$ & $87(n=668)$ & $8.0 \pm 1.5$ & $22 / 2$ & 65 & $48 / 13$ & 85 \\
\hline
\end{tabular}

Social status I = highest, $\mathrm{V}=$ lowest

Values are means \pm SD or percent of patients

${ }^{\text {a }}$ for definiton see Results

Table 2. Insulin therapy and dietary habits in relation to social status

\begin{tabular}{|c|c|c|c|c|c|c|c|c|c|c|}
\hline $\begin{array}{l}\text { Social } \\
\text { status }\end{array}$ & $n$ & $\begin{array}{l}\text { Injections } \\
\text { per day < } 3 / \\
\geq 3 / \mathrm{CSII} \\
\text { (\% pa- } \\
\text { tients) }\end{array}$ & $\begin{array}{l}\text { Previously } \\
\text { recommen- } \\
\text { ded by a } \\
\text { physician } \\
\text { to inject } \\
\text { more of- } \\
\text { ten }{ }^{\text {a }} \% \text { pa- } \\
\text { tients) }\end{array}$ & $\begin{array}{l}\text { Using a } \\
\text { pen device } \\
\text { (\% pa- } \\
\text { tients) }\end{array}$ & $\begin{array}{l}\text { Insulin } \\
\text { dose adap- } \\
\text { tation ac- } \\
\text { cording to } \\
\text { blood glu- } \\
\text { cose values } \\
\text { (\% pa- } \\
\text { tients) }\end{array}$ & $\begin{array}{l}\text { Insulin } \\
\text { dose adap- } \\
\text { tation ac- } \\
\text { cording to } \\
\text { carbohy- } \\
\text { drate inta- } \\
\text { ke (\% pa- } \\
\text { tients) }\end{array}$ & $\begin{array}{l}\text { Following a } \\
\text { meal plan } \\
\text { (\% pa- } \\
\text { tients) }\end{array}$ & $\begin{array}{l}\text { Skipping } \\
\text { main meals } \\
\text { at least } \\
\text { once per } \\
\text { week (\% } \\
\text { patients) }\end{array}$ & $\begin{array}{l}\text { Consuming } \\
\text { sugar nev- } \\
\text { er/several } \\
\text { times per } \\
\text { week or } \\
\text { daily (\% } \\
\text { patients) }\end{array}$ & $\begin{array}{l}\text { Alcohol con- } \\
\text { sumption } \\
\text { (g/week) }\end{array}$ \\
\hline I & 131 & $11 / 74 / 15$ & $64(n=14)$ & $73(n=111)$ & 86 & 77 & 18 & 41 & $21 / 47$ & $57 \pm 71$ \\
\hline II & 141 & $15 / 79 / 6$ & $42(n=21)$ & $76(n=132)$ & 84 & 72 & 23 & 42 & $10 / 50$ & $55 \pm 92$ \\
\hline III & 136 & $19 / 68 / 13$ & $46(n=26)$ & $71(n=119)$ & 82 & 65 & 28 & 33 & $9 / 37$ & $52 \pm 105$ \\
\hline IV & 146 & 21/72/8 & $53(n=30)$ & $70(n=135)$ & 75 & 53 & 36 & 24 & $23 / 37$ & $39 \pm 76$ \\
\hline V & 130 & $39 / 56 / 5$ & $47(n=51)$ & $70(n=124)$ & 63 & 41 & 44 & 24 & 28/31 & $23 \pm 47$ \\
\hline All & 684 & $21 / 70 / 9$ & $49(n=142)$ & $72(n=621)$ & 78 & 62 & 30 & 33 & $18 / 40$ & $45 \pm 82$ \\
\hline
\end{tabular}

Social status $\mathrm{I}=$ highest, $\mathrm{V}=$ lowest

Values are means \pm SD or percent of patients
${ }^{a}$ for patients who use $<3$ injections/day

${ }^{\mathrm{b}}$ for patients not using CSII $(p<0.001$ and $p=0.0075$, respectively, analysis of variance, Table 1). There were no significant associations between social status and gender and body mass index (Table 1). Of 668 patients with measurements of C-peptide 581 (87\%) were C-peptide negative (Table 1).

Metabolic control (Table 1). Mean $\mathrm{HbA}_{1 \mathrm{c}}$ for the total patient group was $8.0 \%$ (range 4.5 to $12.8 \%$ ); $9 \%$ of the patients had an $\mathrm{HbA}_{1 \mathrm{c}}$ value $\leq 6.1 \%$, $39 \% \leq 7.5 \%$ and $22 \%>9 \%$. Lower social status was associated with higher $\mathrm{HbA}_{1 \mathrm{c}}$ values $(p<0.0001)$. Accordingly, there was a tendency of more patients from higher social class reporting at least one mild hypoglycaemic reaction during the preceding week. A total of $325(48 \%)$ patients had a history of severe hypoglycaemia necessitating treatment with glucose i.v. or glucagon injection. For the preceding 12 months $86(13 \%)$ patients reported a total of 141 events of severe hypoglycaemia (incidence 0.21 cases per patient-year). A post-diabetesmanifestation life-time history of ketoacidosis (emer- gency treatment in hospital) was reported by $22 \%$ of the patients, $16(2 \%)$ patients reported a total of 21 cases of ketoacidosis for the preceding 12 months (incidence 0.03 cases per patient-year). There was a clear trend of more lower social class patients experiencing ketoacidosis.

Insulin therapy (Table 2). Mean insulin dosage for all 684 patients was $0.65 \pm 0.22 \mathrm{IU} \cdot \mathrm{kg}^{-1} \cdot$ day $^{-1} ; 70 \%$ used 3 or more insulin injections per day, $9 \%$ used CSII, $49 \%$ injected insulin 4 to 5 times per day, and $9 \%$ injected even more often; 570 patients $(83 \%)$ used regular insulin. There was a strong association between social class and the number of daily insulin injections and the use of CSII $(p<0.0001$, OR 1.62, CI 1.38-1.9). Patients who used less than 3 injections per day were asked whether they had been recommended by a physician to inject insulin more often; $49 \%$ answered "yes", and there was no respective association with social status. In addition, there was no relationship between the usage of a pen device and social status. 
Table 3. Metabolic monitoring in relation to social status

\begin{tabular}{|c|c|c|c|c|c|c|c|c|}
\hline $\begin{array}{l}\text { Social } \\
\text { status }\end{array}$ & $n$ & $\begin{array}{l}\text { Blood glucose } \\
\text { measurements } \\
\text { per day } 0 /<1 / \\
1-2 / \geq 3(\%)\end{array}$ & $\begin{array}{l}\text { Owning a } \\
\text { blood glucose } \\
\text { meter }(\%)\end{array}$ & $\begin{array}{l}\text { Accurate } \\
\text { blood glucose } \\
\text { measurements } \\
(\%)\end{array}$ & $\begin{array}{l}\text { Aware of ma- } \\
\text { terials for mea- } \\
\text { suring aceto- } \\
\text { nuria }(\%)\end{array}$ & $\begin{array}{l}\text { Aware of } \\
\mathrm{HbA}_{1} / \mathrm{HbA}_{1 \mathrm{c}} \\
\text { measurements }^{\mathrm{a}} \\
(\%)\end{array}$ & $\begin{array}{l}\text { Knowing last } \\
\mathrm{HbA}_{1} / \mathrm{HbA}_{1 \mathrm{c}} \\
\operatorname{value}^{\mathrm{b}}(\%)\end{array}$ & 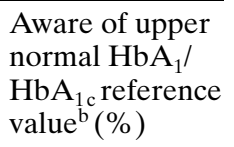 \\
\hline $\mathrm{I}$ & 131 & $1 / 8 / 16 / 76$ & 87 & $87(n=105)$ & 98 & 100 & $89(n=131)$ & $75(n=131)$ \\
\hline II & 141 & $2 / 10 / 16 / 72$ & 87 & $84(n=109)$ & 96 & 99 & $85(n=140)$ & $65(n=140)$ \\
\hline III & 136 & $2 / 15 / 18 / 65$ & 88 & $84(n=108)$ & 93 & 98 & $90(n=132)$ & $64(n=132)$ \\
\hline IV & 146 & $4 / 10 / 16 / 69$ & 91 & $87(n=119)$ & 90 & 93 & $81(n=136)$ & $54(n=135)$ \\
\hline V & 130 & $9 / 17 / 19 / 55$ & 85 & $87(n=97)$ & 82 & 86 & $66(n=113)$ & $38(n=113)$ \\
\hline All & 684 & $4 / 12 / 17 / 68$ & 88 & $86(n=538)$ & 92 & 95 & $83(n=652)$ & $60(n=651)$ \\
\hline
\end{tabular}

Social status $\mathrm{I}=$ highest, $\mathrm{V}=$ lowest

${ }^{a}$ for definiton see results section

${ }^{b}$ for patients aware of $\mathrm{HbA}_{1} / \mathrm{HbA}_{1 \mathrm{c}}$ measurements

Metabolic monitoring (Table 3). Lower social class patients reported performing blood glucose measurements less often than high social class patients $(p=0.0001, \mathrm{OR}=1.38$, CI 1.18-1.61). There was no association between social class and the percentage of patients using a blood glucose meter. The accuracy of blood glucose self-measurements was assessed by comparing the value obtained by the patient using his own method with the laboratory measurement using Reflotron. A difference of less than $20 \%$ between the two results was considered as an acceptable accuracy. There was no association between the percentage of patients with accurate measurements and social status. More high social class patients were aware of methods for self-monitoring of acetonuria. Of the total group $652(95 \%)$ patients knew about $\mathrm{HbA}_{1} /$ $\mathrm{HbA}_{1 \mathrm{c}}$ measurements, i. e. they could roughly explain what they stand for; $83 \%$ of patients reported to know their last value. High social class patients were better informed about their $\mathrm{HbA}_{1} / \mathrm{HbA}_{1 \mathrm{c}}$ measurements than low social class patients.

Insulin dose adaptation (Table 2). Of the 684 patients $532(78 \%)$ reported adapting insulin dosages on a day-to-day basis according to blood glucose self-measurements and $420(62 \%)$ according to varying amounts of carbohydrate intake. More high social class than low social class patients adjusted insulin dosages $(p<0.0001, \mathrm{OR}=1.61$, CI 1.33-1.94 and $p<0.0001$, and $\mathrm{OR}=1.73$, CI 1.46-2.05, respectively).

Nutritional habits (Table 2). Of the total group 30\% reported using a meal plan; fewer high social class patients used a meal plan $(p<0.0001$, OR 0.69 , CI $0.58-0.81) ; 33 \%$ reported skipping main meals at least once per week; more high social class patients reported skipping main meals; there was a trend of more high social class patients reporting consuming sugar and sugar-containing nutrients on a regular basis; self-reported alcohol consumption for the preceding week was significantly higher among high social class patients.
Diabetes education (Table 4). Of the total patient group $62 \%$ reported that they had participated in a 5-12 day structured inpatient DTTP for groups of up to 10 patients (delivered by a diabetes educator according to a curriculum). Higher social status was associated with a higher percentage of patients who had participated in a DTTP $(p=0.0013$, OR $=1.29$, CI 1.10-1.50). Of the 261 patients who had never participated in a DTTP $34 \%$ reported that they had previously been recommended by a physician to participate in such a DTTP, but there was no association with social class ( $p=0.69$, OR 0.95 , CI $0.74-1.23)$. Of the 261 patients who had not participated in a DTTP $43 \%$ reported having received diabetes education including some kind of training in insulin dose adaptation (e.g. individual counselling, education during a stay in a so-called diabetes rehabilitation hospital). Thus, a total of $535(78 \%)$ patients received some kind of training in insulin dose adaptation.

Specialized outpatient care (Table 4). Of the 684 patients $50 \%$ reported having consulted a diabetologist on an outpatient basis or had visited a specialized diabetes outpatient clinic at some time and $27 \%$ had done so during the preceding year. Higher social class was associated with a higher percentage of patients who had consulted a specialized diabetes outpatient clinic at some time $(p<0.0001, \quad$ OR $=1.36$, CI 1.17-1.56). Of the patients who had never consulted a diabetologist $28 \%$ reported that they had previously been recommended by a physician to do so, but there was no association with social status $(p=0.83$, OR 1.03, CI 0.81-1.29).

Family physician contacts (Table 4). 583 patients were asked about the number of consultations in their family physician's office during the preceding year. Patients of higher social class reported fewer consultations than patients of lower social class $(p=0.0039$, OR 0.80, CI 0.69-0.93). 
Table 4. Specialized diabetes care, family physician contacts, and membership of patient associations in relation to social status

\begin{tabular}{|c|c|c|c|c|c|c|c|c|}
\hline $\begin{array}{l}\text { Social } \\
\text { status }\end{array}$ & $n$ & $\begin{array}{l}\text { Participation } \\
\text { in a DTTP } \\
\text { (\% patients) }\end{array}$ & $\begin{array}{l}\text { Previously re- } \\
\text { commended by } \\
\text { a physician to } \\
\text { participate in a } \\
\text { DTTP } \\
\text { (\% patients) }\end{array}$ & $\begin{array}{l}\text { Education ab- } \\
\text { out insulin } \\
\text { dose adapta- } \\
\text { tion outside a } \\
\text { DTTPa } \\
\text { (\% patients) }\end{array}$ & $\begin{array}{l}\text { Visit in a dia- } \\
\text { betes out-pa- } \\
\text { tient clinic } \\
\text { (\% patients) }\end{array}$ & $\begin{array}{l}\text { Previously re- } \\
\text { commended by a } \\
\text { physician to visit } \\
\text { a diabetes out- } \\
\text { patient clinic }{ }^{\mathrm{b}} \\
\text { (\% patients) }\end{array}$ & $\begin{array}{l}\text { Family physician } \\
\text { contacts during } \\
\text { preceding year } \\
<5 / 5-8 />8 \\
\text { (\% patients) }\end{array}$ & $\begin{array}{l}\text { Member of } \\
\text { patient asso- } \\
\text { ciation } \\
\text { (\% patients) }\end{array}$ \\
\hline I & 131 & 69 & $34(n=41)$ & $54(n=41)$ & 63 & $29(n=49)$ & $28 / 23 / 48(n=124)$ & 31 \\
\hline II & 141 & 72 & $41(n=39)$ & $51(n=39)$ & 54 & $26(n=65)$ & $20 / 23 / 57(n=114)$ & 16 \\
\hline III & 136 & 58 & $32(n=57)$ & $49(n=57)$ & 47 & $29(n=72)$ & $15 / 27 / 58(n=110)$ & 19 \\
\hline IV & 146 & 57 & $30(n=63)$ & $38(n=63)$ & 47 & $32(n=78)$ & $18 / 24 / 58(n=126)$ & 15 \\
\hline V & 130 & 52 & $34(n=61)$ & $31(n=61)$ & 38 & $23(n=81)$ & $16 / 18 / 66(n=109)$ & 11 \\
\hline All & 684 & 62 & $34(n=261)$ & $43(n=261)$ & 50 & $28(n=345)$ & $20 / 23 / 57(n=583)$ & 18 \\
\hline
\end{tabular}

Social status I = highest, $\mathrm{V}=$ lowest

${ }^{a}$ for patients who had never participated in a DTTP

${ }^{\mathrm{b}}$ for patients who had never visited a diabetes out-patient clinic

Table 5. Blood pressure and smoking in relation to social status

\begin{tabular}{|c|c|c|c|c|c|c|c|c|c|}
\hline $\begin{array}{l}\text { Social } \\
\text { status }\end{array}$ & $n$ & $\begin{array}{l}\text { Blood pressure mea- } \\
\text { surements by the } \\
\text { family physician } \\
\text { during preceding } \\
\text { year } 0 / 1-4 />4 \\
\text { (\% patients) }\end{array}$ & $\begin{array}{l}\text { Aware of hy- } \\
\text { pertension for } \\
\text { all patients/for } \\
\text { patients with } \\
\text { hypertension } \\
\text { (\% patients) }\end{array}$ & $\begin{array}{l}\text { Drug } \\
\text { treatment } \\
\text { for hyper- } \\
\text { tension } \\
\text { (\% pa- } \\
\text { tients) }\end{array}$ & $\begin{array}{l}\text { Blood pres- } \\
\text { sure systolic/ } \\
\text { diastolic } \\
(\mathrm{mm} \mathrm{Hg})\end{array}$ & $\begin{array}{l}\text { Blood pres- } \\
\text { sure control } \\
\text { level } 1 / 2 / 3^{b} \\
\text { (\% patients) }\end{array}$ & $\begin{array}{l}\text { Blood } \\
\text { pressure } \\
\text { self moni- } \\
\text { toring (\% } \\
\text { patients) }\end{array}$ & $\begin{array}{l}\text { Non-smo- } \\
\text { kers/ex-smo- } \\
\text { kers/smokers } \\
\text { (\% patients) }\end{array}$ & $\begin{array}{l}\text { Cigarette } \\
\text { pack years }\end{array}$ \\
\hline $\bar{I}$ & 131 & $18 / 64 / 18(n=131)$ & $18 / 47(n=49)$ & 16 & $136 / 76 \pm 17 / 10$ & $62 / 27 / 11$ & 34 & $55 / 19 / 26$ & $6 \pm 10$ \\
\hline II & 141 & $24 / 56 / 20(n=140)$ & $15 / 53(n=40)$ & 11 & $133 / 74 \pm 19 / 12$ & $72 / 18 / 10$ & 26 & $42 / 13 / 45$ & $8 \pm 11$ \\
\hline III & 136 & $18 / 47 / 35(n=135)$ & $24 / 52(n=56)$ & 18 & $137 / 77 \pm 21 / 11$ & $64 / 21 / 15$ & 30 & $35 / 23 / 43$ & $11 \pm 15$ \\
\hline IV & 146 & $21 / 49 / 31(n=146)$ & $23 / 48(n=67)$ & 19 & $139 / 75 \pm 21 / 12$ & $54 / 26 / 20$ & 34 & $38 / 15 / 47$ & $11 \pm 13$ \\
\hline $\mathrm{V}$ & 130 & $16 / 38 / 47(n=129)$ & $28 / 60(n=58)$ & 22 & $139 / 77 \pm 26 / 12$ & $56 / 19 / 25$ & 24 & $32 / 20 / 48$ & $14 \pm 19$ \\
\hline All & 684 & $19 / 51 / 30(n=681)$ & $21 / 52(n=270)$ & 17 & $137 / 76 \pm 21 / 11$ & $62 / 22 / 16$ & 30 & $40 / 18 / 42$ & $10 \pm 14$ \\
\hline
\end{tabular}

Social status $\mathrm{I}=$ highest, $\mathrm{V}=$ lowest

${ }^{\mathrm{b}}$ level $1=$ systolic $<140$ and diastolic $<90$, level $2=\geq 140$

Values are means \pm SD or percent of patients

${ }^{\text {a }}$ systolic $\geq 140$ or diastolic $\geq 90 \mathrm{~mm} \mathrm{Hg}$ or drug treatment for

$<160$ or $\geq 90<95$, level $3=\geq 160$ or $\geq 95 \mathrm{~mm} \mathrm{Hg}$

hypertension

Member of self help group or diabetes association (Table 4). Fewer patients of low social class were members of self-help groups or a diabetic patient association.

Blood pressure (Table 5). Of the 684 patients 147 $(21 \%)$ reported having hypertension, and 202 $(30 \%)$ reported measuring blood pressure themselves at least sometimes; of the 147 patients with known hypertension $96(65 \%)$ performed blood pressure self monitoring; $81 \%$ of all patients reported having had at least one blood pressure measurement in the family physician's office during the preceding 12 months; 118 (17\%) of patients reported taking antihypertensive drugs (one patient without known hypertension). There was a tendency towards more patients of the lowest social class undergoing more blood pressure measurements by the family physician and more of them being aware of having hypertension (Table 5). Of the 536 patients without known hypertension $29(5.4 \%)$ had blood pressure values $160 \mathrm{mmHg}$ or more systolic or $95 \mathrm{mmHg}$ or more diastolic. Of the 147 patients with known hypertension $117(80 \%)$ were being treated with antihypertensive drugs, and $30(20 \%)$ of them had normal blood pressure values $(<140 / 90 \mathrm{mmHg})$, whereas $82(56 \%)$ had blood pressure values $160 \mathrm{mmHg}$ or more systolic or $95 \mathrm{mmHg}$ or more diastolic. There were significant differences with respect to blood pressure control across social class levels. Fewer patients of low social class had blood pressure values under 140/ $90 \mathrm{mmHg}$ and more had uncontrolled blood pressure $(\geq 160 / 95 \mathrm{mmHg})(p=0.049$; OR 0.81, CI 0.66-0.99).

Smoking (Table 5). Of the 282 women 100 (35\%) and of the 402 men 186 (46\%) were smokers. High social class patients were less likely to be smokers $(p=0.0002$, OR 0.74, CI 0.64-0.87) and had fewer cigarette pack years $(p<0.001)$.

Diabetic nephropathy (Table 6). Of the total patient group $14 \%$ knew that they had diabetic renal complications, $69 \%$ were aware that proteinuria/albuminuria had been measured at some time, whereas $27 \%$ were not aware that such diagnostic tests exist. For the group of patients with 'at least microalbuminuria' (combined nephropathy levels 2 to $5, n=225$ ) there was no significant difference between awareness and 
Table 6. Patient awareness of diagnosed complications and performed screening examinations in relation to social status

\begin{tabular}{|c|c|c|c|c|c|c|c|c|}
\hline $\begin{array}{l}\text { Social } \\
\text { status }\end{array}$ & $n$ & $\begin{array}{l}\text { Aware of nephro- } \\
\text { pathy for all pati- } \\
\text { ents/for patients } \\
\text { with at least mi- } \\
\text { croproteinuria } \\
\text { (\% patients) }\end{array}$ & $\begin{array}{l}\text { Aware of pro- } \\
\text { teinuria/albu- } \\
\text { minuria mea- } \\
\text { surements } \\
\text { (\% patients) }\end{array}$ & $\begin{array}{l}\text { Aware of eye } \\
\text { complications for } \\
\text { all patients/for } \\
\text { patients with any } \\
\text { retinopathy (\% } \\
\text { patients) }\end{array}$ & $\begin{array}{l}\text { Examination } \\
\text { of the retina } \\
\text { during prece- } \\
\text { ding year } \\
\text { (\% patients) }\end{array}$ & $\begin{array}{l}\text { Aware of neu- } \\
\text { rologic foot } \\
\text { complications } \\
\text { (\% patients) }\end{array}$ & $\begin{array}{l}\text { Examination } \\
\text { of the feet du- } \\
\text { ring preceding } \\
\text { year (\% pa- } \\
\text { tients) }\end{array}$ & $\begin{array}{l}\text { Examination } \\
\text { with the tuning } \\
\text { fork at any time } \\
\text { (\% patients) }\end{array}$ \\
\hline I & 131 & $12 / 45(n=29)$ & 77 & $38 / 77(n=60)$ & 83 & 13 & 41 & $86(n=130)$ \\
\hline II & 141 & $10 / 29(n=38)$ & 73 & $31 / 70(n=54)$ & 77 & 12 & 38 & $81(n=139)$ \\
\hline III & 136 & $18 / 43(n=44)$ & 68 & $40 / 84(n=64)$ & 83 & 20 & 39 & $82(n=132)$ \\
\hline IV & 146 & $12 / 32(n=57)$ & 70 & $36 / 65(n=72)$ & 76 & 17 & 39 & $76(n=143)$ \\
\hline V & 130 & $17 / 28(n=57)$ & 57 & $49 / 78(n=73)$ & 79 & 26 & 45 & $78(n=125)$ \\
\hline All & 684 & $14 / 34(n=225)$ & 69 & $39 / 75(n=323)$ & 80 & 18 & 40 & $81(n=669)$ \\
\hline
\end{tabular}

Social status I = highest, $\mathrm{V}=$ lowest

Table 7. Micro- and macrovascular complications, drug therapy, and hospital days in relation to social status

\begin{tabular}{|c|c|c|c|c|c|c|c|}
\hline $\begin{array}{l}\text { Social } \\
\text { status }\end{array}$ & $n$ & $\begin{array}{l}\text { Nephropathy levels } \\
1 / 2 / 3 / 4 / 5^{\mathrm{a}} \text { (\% patients) }\end{array}$ & $\begin{array}{l}\text { Retinopathy } \\
\text { levels } 1 / 2 / 3 / 4^{\mathrm{a}} \\
\text { (\% patients) }\end{array}$ & $\begin{array}{l}\text { Foot complica- } \\
\text { tions levels } 1 / 2 / 3^{a} \\
\text { (\% patients) }\end{array}$ & $\begin{array}{l}\text { Macrovascular } \\
\text { complications } \\
\text { (\% patients) }\end{array}$ & $\begin{array}{l}\text { Drug therapy } \\
\text { (except antihy- } \\
\text { pertensive drugs) } \\
\text { (\% patients) }\end{array}$ & $\begin{array}{l}\text { Hospital days per } \\
\text { patient during } \\
\text { preceding year }\end{array}$ \\
\hline I & 131 & $78 / 15 / 5 / 0 / 2(n=131)$ & $54 / 25 / 18 / 2(n=130)$ & $86 / 8 / 5(n=131)$ & 5 & 24 & $2 \pm 8$ \\
\hline II & 141 & $73 / 21 / 4 / 0 / 2(n=141)$ & $61 / 27 / 9 / 3(n=139)$ & $944 / 1(n=141)$ & 1 & 24 & $5 \pm 12$ \\
\hline III & 136 & $67 / 18 / 8 / 3 / 4(n=135)$ & $52 / 20 / 22 / 5(n=134)$ & $83 / 11 / 6(n=136)$ & 4 & 32 & $7 \pm 18$ \\
\hline IV & 146 & $61 / 25 / 5 / 4 / 4(n=146)$ & $50 / 24 / 19 / 7(n=143)$ & $84 / 12 / 4(n=143)$ & 8 & 42 & $9 \pm 28$ \\
\hline V & 130 & $55 / 24 / 13 / 5 / 2(n=128)$ & $42 / 26 / 25 / 6(n=126)$ & $63 / 25 / 11(n=126)$ & 19 & 47 & $8 \pm 16$ \\
\hline All & 684 & $67 / 21 / 7 / 2 / 3(n=681)$ & $52 / 25 / 19 / 5(n=672)$ & $83 / 12 / 5(n=677)$ & 7 & 34 & $6 \pm 18$ \\
\hline
\end{tabular}

Social status I = highest, $\mathrm{V}=$ lowest

Values are means \pm SD or percent of patients
${ }^{a}$ for definition see methods section

${ }^{\mathrm{b}}$ for definition see results section social status. The distribution of patients with respect to levels of nephropathy is shown in Table 7. Of the 19 patients on renal replacement therapy, 11 were undergoing haemodialysis, 4 continuous ambulatory peritoneal dialysis and 4 had a renal transplant. The proportional odds model could not be used to describe the association between levels of nephropathy and social status. Instead, binary logistic regression analyses were performed. Using the response variable 'at least microproteinuria' (combined levels 2 to $5, n=225)$ compared with patients with normal proteinuria (level $1, n=456$ ) and using the response variable 'at least macroproteinuria' (combined levels 3 to $5, n=83$ ) compared with combined levels 1 and 2 $(n=598)$ higher social status was associated with a lower risk of nephropathy (Tables 8 and 9). In order to assess to what extent the association between social class and nephropathy could be explained by established risk factors for nephropathy the same logistic regressions were performed, with additionally adjusting for $\mathrm{HbA}_{1 \mathrm{c}}$, systolic and diastolic blood pressure and smoking status (cigarette pack years). In these models, the association between social status and the response variables was attenuated but still statistically significant (Tables 8 and 9).
Diabetic retinopathy (Table 6). Of the total patient group 264 (39\%) reported having diabetic eye complications. On examination, $8 \%$ of these 264 patients had a normal fundus, whereas $20 \%$ of the 410 patients who reported not being aware of complications had some retinopathy (76 patients level 1,5 patients level 2). Of the total group, $80 \%$ reported having had an eye examination to screen for diabetic complications during the preceding year; $81 \%$ of these patients reported that the last examination had been performed after dilation of the pupils, an additional $4 \%$ had a photograph of the retina without dilation of the pupils. There was no association between social status and awareness of eye complications. The frequencies of the various levels of retinopathy in the total patient group and across the various levels of social class are shown in Table 7. There was a significantly lower risk of retinopathy for higher social class patients, an association which was, however, no longer statistically significant after adjustment for established risk factors for retinopathy (Table 10).

Foot complications (Tables 6 and 7). Of the total patient group $120(18 \%)$ reported having diabetic neurologic foot complications, $81 \%$ remembered having had a foot examination with the tuning fork at some time, but $51 \%$ did not remember having had any foot examination to screen for diabetic complications 
Table 8. Binary logistic regression for nephropathy (at least microproteinuria)

\begin{tabular}{|c|c|c|c|c|c|}
\hline Variables & Logistic coefficient & Standard error & $p$-value & $\begin{array}{l}\text { Difference for } \\
\text { odds ratio }\end{array}$ & $\begin{array}{l}\text { Odds ratio } \\
(95 \% \mathrm{CI})\end{array}$ \\
\hline \multicolumn{6}{|c|}{$\begin{array}{l}\text { Adjustment for age }^{2}, \text { diabetes duration } \\
\text { (Hosmer and Lemeshow goodness-of-fit } p=0.378 \text { ) }\end{array}$} \\
\hline Intercept 1 & -0.2024 & 0.0257 & 0.4312 & - & - \\
\hline Social status ${ }^{\mathrm{a}}$ & -0.0661 & 0.0167 & 0.0001 & 1 level & $0.719(0.610-0.846)$ \\
\hline $\mathrm{Age}^{2}$ (years) & -0.0003 & 0.0002 & 0.0315 & - & - \\
\hline Diabetes duration $^{2}$ (years) & -0.0015 & 0.0003 & 0.0001 & - & - \\
\hline \multicolumn{6}{|c|}{$\begin{array}{l}\text { Additional adjustment for } \mathrm{HbA}_{1 \mathrm{c}} \text {, blood pressure, smoking } \\
\text { (Hosmer and Lemeshow goodness-of-fit } p=0.10 \text { ) }\end{array}$} \\
\hline Intercept 1 & -5.6681 & 0.8902 & 0.0001 & - & - \\
\hline Social status ${ }^{\mathrm{a}}$ & -0.0474 & 0.0178 & 0.0078 & 1 level & $0.789(0.663-0.939)$ \\
\hline $\operatorname{Age}^{2}$ (years) & -0.0007 & 0.0002 & 0.0001 & - & - \\
\hline Diabetes duration $^{2}$ (years) & 0.0016 & 0.0003 & 0.0001 & - & - \\
\hline $\mathrm{HbA}_{1 \mathrm{c}}(\%)$ & 0.2132 & 0.0622 & 0.0006 & $1 \%$ & $1.238(1.096-1.398)$ \\
\hline Systolic blood pressure $(\mathrm{mm} \mathrm{Hg})$ & 0.0269 & 0.0050 & 0.0001 & $5 \mathrm{~mm} \mathrm{Hg}$ & $1.144(1.089-1.202)$ \\
\hline Cigarette pack years & 0.0242 & 0.0070 & 0.0005 & 10 pack years & $1.274(1.112-1.460)$ \\
\hline
\end{tabular}

${ }^{a}$ Higher social class levels are associated with a lower risk of nephropathy (at least microproteinuria)

Table 9. Binary logistic regression for nephropathy (at least macroproteinuria)

\begin{tabular}{|c|c|c|c|c|c|}
\hline Variables & Logistic coefficient & Standard error & $p$-value & $\begin{array}{l}\text { Difference for } \\
\text { odds ratio }\end{array}$ & $\begin{array}{l}\text { Odds ratio } \\
(95 \% \mathrm{CI})\end{array}$ \\
\hline \multicolumn{6}{|c|}{$\begin{array}{l}\text { Adjustment for age }{ }^{2} \text {, diabetes duration, diabetes duration } \\
\text { (Hosmer and Lemeshow goodness-of-fit } p=0.68 \text { ) }\end{array}$} \\
\hline Intercept 1 & -5.6231 & 0.9331 & 0.0001 & - & - \\
\hline Social status ${ }^{\mathrm{a}}$ & -0.0892 & 0.0254 & 0.0005 & 1 level & $0.640(0.499-0.821)$ \\
\hline $\mathrm{Age}^{2}$ (years) & -0.0003 & 0.0002 & 0.0990 & - & - \\
\hline Diabetes duration (years) & 0.3625 & 0.0682 & 0.0001 & - & - \\
\hline Diabetes duration $^{2}$ (years) & -0.0050 & 0.0013 & 0.0001 & - & - \\
\hline \multicolumn{6}{|c|}{$\begin{array}{l}\text { Additional adjustment for } \mathrm{HbA}_{1 \mathrm{c}} \text {, blood pressure, smoking } \\
\text { (Hosmer and Lemeshow goodness-of-fit } p=0.407 \text { ) }\end{array}$} \\
\hline Intercept 1 & -15.9398 & 1.9339 & 0.0001 & - & - \\
\hline Social status ${ }^{\mathrm{a}}$ & -0.0674 & 0.0286 & 0.0186 & 1 level & $0.714(0.539-0.945)$ \\
\hline $\mathrm{Age}^{2}($ years $)$ & -0.0008 & 0.0002 & 0.0020 & - & - \\
\hline Diabetes duration (years) & 0.3610 & 0.0751 & 0.0001 & - & - \\
\hline Diabetes duration ${ }^{2}$ (years) & -0.0049 & 0.0014 & 0.0003 & - & - \\
\hline $\mathrm{HbA}_{1 \mathrm{c}}(\%)$ & 0.4251 & 0.1091 & 0.0001 & $1 \%$ & $1.530(1.235-1.894)$ \\
\hline Systolic blood pressure $(\mathrm{mm} \mathrm{Hg})$ & 0.0493 & 0.0074 & 0.0001 & $5 \mathrm{~mm} \mathrm{Hg}$ & $1.280(1.190-1.376)$ \\
\hline Cigarette pack years & 0.0009 & 0.0090 & 0.9207 & 10 pack years & $1.009(0.845-1.205)$ \\
\hline
\end{tabular}

${ }^{a}$ Higher social class levels are associated with a lower risk of nephropathy (at least macroproteinuria)

outside a hospital stay; during the preceding 12 months $40 \%$ underwent a foot examination (42\% of the 511 patients with a diabetes duration of more than 10 years) with a trend of more patients of low social class being aware of diabetic (neurologic) foot complications. There was no association between patients who had an examination of the feet during the preceding year and social status and no relationship between social status and a foot examination using the tuning fork. Foot pulses could not be assessed in 11 patients (amputation, oedema etc.), and were palpable on both sides (dorsalis pedis or tibialis posterior) in $639(95 \%)$ of the 672 remaining patients. Impaired vibration sensation with a tuning fork score $4 / 8$ or less at the first metatarsal was found in 76 $(13 \%)$ of 668 patients bilaterally and in an additional 29 (4\%) unilaterally. A total of 17 patients (5 women) had a total of 27 amputations of the lower extremities (15 below ankle, 9 below knee, 3 above knee). A total of 27 healed ulcers were reported by $23(3 \%)$ patients, a total of 13 acute ulcers were present in 12 $(2 \%)$ patients. A total of $33(4.8 \%)$ patients had a healed or acute ulcer and $37(5.4 \%)$ had a healed or acute ulcer or an amputation. Fewer patients of high social status had foot complications $(p=0.001$, OR 0.69 , CI 0.55-0.86).

Macrovascular complications (Table 7). 23 (3.4\%) patients had a history of angina pectoris, 18 (2.6\%) patients had survived a myocardial infarction; coronary bypass surgery or angioplasty was reported by $13(1.9 \%)$ patients; $11(1.6 \%)$ patients reported having had a cerebral infarction (ischaemia duration for more than $24 \mathrm{~h}$ ), claudicatio intermittens was report- 
Table 10. Proportional odds model for retinopathy (3 levels $\left.{ }^{a}\right)$

\begin{tabular}{|c|c|c|c|c|c|}
\hline Variables & Logistic coefficient & Standard error & $p$-value & $\begin{array}{l}\text { Difference for } \\
\text { odds ratio }\end{array}$ & $\begin{array}{l}\text { Odds ratio } \\
(95 \% \mathrm{CI})\end{array}$ \\
\hline \multicolumn{6}{|c|}{$\begin{array}{l}\text { Adjustment for age, age }^{2}, \text { diabetes duration, diabetes duration } \\
\text { (score test for the proportional odds assumption } p=0.736 \text { ) }\end{array}$} \\
\hline Intercept 1 & -8.6635 & 1.0370 & 0.0001 & - & - \\
\hline Intercept 2 & -6.9568 & 1.0127 & 0.0001 & - & - \\
\hline Social status ${ }^{\mathrm{b}}$ & -0.0475 & 0.0162 & 0.0034 & 1 level & $0.789(0.673-0.924)$ \\
\hline Age (years) & 0.1581 & 0.0527 & 0.0027 & - & - \\
\hline $\mathrm{Age}^{2}$ (years) & -0.0020 & 0.0006 & 0.0020 & - & - \\
\hline Diabetes duration (years) & 0.3390 & 0.0392 & 0.0001 & - & - \\
\hline Diabetes duration $^{2}$ (years) & -0.0040 & 0.0080 & 0.0001 & - & - \\
\hline \multicolumn{6}{|c|}{$\begin{array}{l}\text { Additional adjustment for } \mathrm{HbA}_{1 \mathrm{c}} \text {, blood pressure, smoking } \\
\text { (score test for the proportional odds assumption } p=0.846 \text { ) }\end{array}$} \\
\hline Intercept 1 & -13.4630 & 1.3967 & 0.0001 & - & - \\
\hline Intercept 2 & -11.6519 & 1.3659 & 0.0001 & - & - \\
\hline Social status ${ }^{\mathrm{b}}$ & -0.0295 & 0.0170 & 0.0825 & 1 level & $0.863(0.730-1.019)$ \\
\hline Age (years) & 0.1359 & 0.0556 & 0.0146 & - & - \\
\hline $\operatorname{Age}^{2}$ (years) & -0.00185 & 0.0007 & 0.0056 & - & - \\
\hline Diabetes duration (years) & 0.3467 & 0.0407 & 0.0001 & - & - \\
\hline Diabetes duration $^{2}$ (years) & -0.0015 & 0.0008 & 0.0001 & - & - \\
\hline $\mathrm{HbA}_{1 \mathrm{c}}(\%)$ & 0.3023 & 0.0650 & 0.0001 & $1 \%$ & $1.353(1.191-1.537)$ \\
\hline Systolic blood pressure $(\mathrm{mm} \mathrm{Hg})$ & 0.0183 & 0.0047 & 0.0001 & $5 \mathrm{~mm} \mathrm{Hg}$ & $1.096(1.047-1.147)$ \\
\hline Cigarette pack years & 0.00477 & 0.0066 & 0.4689 & 10 pack years & $1.049(0.922-1.193)$ \\
\hline
\end{tabular}

${ }^{a}$ retinopathy level 1 , level 2 and combined levels 3 and 4

${ }^{\mathrm{b}}$ Higher social class levels are associated with a lower risk of retinopathy

ed by 30 (4.4\% ) patients, and a peripheral bypass operation or angioplasty was reported by $14(2 \%)$ patients. In total, $50(7.3 \%)$ patients (14 women) had at least one of the mentioned clinically manifest macrovascular complications. As expected, there was a strong relationship between the percentage of patients with macrovascular complications and the level of nephropathy (level 1: 4\%, level 2: $8 \%$, level 3: $13 \%$, level 4: $29 \%$, level 5: $47 \%$ ). Fewer patients of high social class had macrovascular complications $(p=0.0013$, OR 0.56, CI 0.739-0.79).

Time spent in hospital (Table 7). There was an inverse association between number of days in hospital during the preceding year and social status.

Drug therapy. Apart from antihypertensive drug therapy (Table 5), 231 (34\%) patients were taking at least one drug on a regular basis. About twice as many patients of the lowest social class were taking drugs compared to patients of the highest social class (Table 7).

Quality of life. Detailed results have been published elsewhere [20]. Social status was significantly associated with physical complaints $(p<0.001)$, diet restrictions $(p<0.001)$ and worries about future $(p<0.05)$, i.e. quality of life scores improved with increasing social status. When accounting for $\mathrm{HbA}_{1 \mathrm{c}}$ within the regression model, the statistical asssociations between social status and the subscales physical complaints and diet restrictions were still significant $(p<0.001)$, whereas worries about the future were no longer sig- nificantly associated with social status. Social status was not significantly associated with the total PWTSS, but there were relevant associations between social status and patients' preferences for certain treatment goals. Thus, more patients with a lower social status were aiming for normal and stable blood glucose values, but at the same time were striving more intensively to avoid even mild hypoglycaemia and to avoid blood glucose self-monitoring $(p<0.005)$. Regarding treatment satisfaction, patients with a lower social class were less satisfied with their flexibility during leisure time $(p<0.01)$, but more satisfied with their perceived protection against late complications $(p<0.05)$ despite higher $\mathrm{HbA}_{1 \mathrm{c}}$-levels $\left(r=0.19\right.$ for social status and $\mathrm{HbA}_{1 \mathrm{c}}$, $p<0.001)$.

\section{Discussion}

This study presents, for the first time, a comprehensive assessment of the quality of care of adult persons with Type I diabetes under the conditions of the availability of modern approaches of intensified treatment on a population-based basis. The results show that in North-Rhine (approximately 9.5 million inhabitants) the achieved standards of diabetes care are high with respect to the implementation of intensified treatment regimens, the level of patient education achieved and treatment control by the physicians (measurements of $\mathrm{HbA}_{1 \mathrm{c}}$ ). The achieved degree of metabolic control with a mean $\mathrm{HbA}_{1 \mathrm{c}}$ of $8.0 \%$ and an incidence of severe hypoglycaemia of 0.21 cases 
per patient-year in a population with a mean diabetes duration of 18 years compares favourably with other studies [21-23]. In the 10-year follow-up of the Wisconsin study during 1990-1992, mean $\mathrm{HbA}_{1 \mathrm{c}}$ was $9.4 \%$, only $23 \%$ of patients used 3 or more insulin injections per day, $1 \%$ used CSII, but $2 \%$ had a pancreas transplant. In the present study, about $70 \%$ used 3 or more insulin injections and 9\% CSII.

Improvement of care appears, however, necessary with respect to the quality of blood pressure control. Only $27 \%$ of patients on antihypertensive drug therapy had normal blood pressure values $(<140 /$ $90 \mathrm{mmHg}$ ), whereas as many as $70 \%$ had uncontrolled hypertension $(\geq 160 / 95 \mathrm{mmHg})$. These findings are in accordance with our previous reports that suggested that blood pressure control in diabetes outside clinical trials is a neglected area $[12,24]$. A comprehensive hypertension treatment and teaching programme as part of specialized outpatient care appears necessary in order to achieve optimal blood pressure control in Type I diabetes [12]. It has been shown that such an integrative approach of care of patients with late complications can substantially retard the manifestation of renal insufficiency and reduce mortality [25]. The high level of uncontrolled hypertension and the low level of patient awareness of measurements of proteinuria or albuminuria in the present population-based study underscore the need for specialized care for Type I diabetic patients with late complications.

A further area for improvement of care as identified in this study is the prevention of foot complications. Only $42 \%$ of patients with a diabetes duration of more than 10 years remembered having undergone foot examination during the preceding 12 months, and $51 \%$ of patients could not remember having ever had any foot examination outside a hospital stay. The high proportion of $81 \%$ of patients reporting having had an examination with the tuning fork is obviously due to appropriate medical care during hospital stays.

Overall health status of low social class patients was worse than that of high social class patients as reflected by a higher rate of micro- and macrovascular complications, more patients using pharmacological medication and more hospitalisation days. In accordance with the study hypothesis there was a significant association between social class and late complications. Clinically overt nephropathy and foot complications were more than three times and retinopathy almost twice as prevalent among patients of the lowest social class compared with those of the highest social class. This association was partly explained by a lower control of risk factors. Thus, lower social class patients had higher $\mathrm{HbA}_{1 \mathrm{c}}$ levels, a lower degree of blood pressure control and more of them were smokers. However, it should be stressed that even patients of the lowest quintile of social class had a mean
$\mathrm{HbA}_{1 \mathrm{c}}$ level of $8.4 \%$ which is still substantially lower than the $9.4 \%$ for the total Wisconsin Type I diabetic population [21].

In accordance with these findings and with our pre-study assumptions, treatment strategies were less effective in lower social class patients. Thus, fewer lower social class patients had intensified insulin substitution regimens, they measured blood glucose less often, fewer of them adapted insulin dosages on a day-to-day basis, and fewer had participated in a DTTP or had consulted a diabetologist on an outpatient basis. However, in contrast to our pre-study assumptions, these differences were not due to a lower standard of care provided by their family physicians and the health care system in general. Thus, there were no significant differences across social class levels with respect to the percentage of patients who had been recommended by their physicians to inject insulin more often, to participate in a DTTP or visit a diabetes clinic. In addition, diagnostic screening tests for late complications were performed to a similar extent in all patients irrespective of social class, if anything, there was even a trend towards more physician contacts and medical examinations in lower social class patients. In addition, in contrast to the study hypothesis, low social class patients were equally well aware of their late complications, and there was no difference with respect to the provision of these patients with modern treatment facilities such as pendevices or blood glucose meters.

Diabetes-related quality of life was lower in low social class patients. Interestingly, low social class patients showed a tendency to have unrealistic treatment goals. They were striving for low blood glucose values even more intensively than higher social class patients, but were less ready to accept potential disadvantages of achieving good glycaemic control such as mild hypoglycaemia and frequent blood glucose self-monitoring. If patients of lower social class have the same access to modern insulin therapy and diabetes care as higher social class patients, but less treatment success, their specific preferences for certain treatment goals reflect important deficits in health motivation. In addition, if these patients feel more protected against late complications despite higher $\mathrm{HbA}_{1 \mathrm{c}}$-levels they might prefer coping strategies relying on unrealistic optimism.

Possible limitations of the study are the following: there is no possibility in the German legal system with its exceptionally strict personal data protection system to definitely prove the representativeness of the patients recruited. However, as has been described in detail elsewhere, several observations indicate that the recruited patients are indeed representative of the general population of Type I diabetic patients in North-Rhine, Germany [11]: the estimated numbers of adults with Type I diabetes in this area are in accordance with the actually available number 
of subjects from the approximately $10 \%$ of recruited family physician practices in North-Rhine. Overall, physician compliance was high, and $73 \%$ of all available patients could be examined. In contrast to previous tertiary care centre based studies $[13,22]$ with approximately equal numbers of male and female subjects, in the present population-based study, the proportion of men was as high as $59 \%$. This figure is in accordance with population based diabetes registers of the former GDR (East Germany) [26] and of Scandinavia [27]. Usually, women show a higher participation in studies on health issues than men. In fact, in the present study, the proportion of men was even somewhat higher in the group of subjects who could not be recruited in comparison to those who participated [11]. Mentally disabled patients (approximately $5 \%)$, non-German citizens $(5.4 \%)$ and patients living in nursing homes were not excluded from the present study. However, in comparison to the general population of North-Rhine, there appeared to be fewer non-German citizens and there was a trend towards a somewhat higher educational level among the diabetic subjects, although statistical analysis was not possible due to different methods of assessment and classification of social class parameters between the present study and the data available for the general population of North-Rhine which had been obtained in the context of a 1993 microcensus [10]. The difference in age between the patients of the present study and the general population of North-Rhine (age groups 18-75 years) of approximately 8.5 years reflects the persisting lower life expectancy of Type I diabetic patients [10].

The design of the present study was cross-sectional. While we have shown that risk factor adjustment attenuates the association between social status and nephropathy and retinopathy, respectively, it is likely that a single measurement of these risk factors at one point does not adequately represent exposure over the life course. Patients who manifest vision impairing retinopathy or overt nephropathy may improve metabolic and blood pressure control and stop smoking. Therefore, given the biologic variability and the errors associated with measurement of these risk factors on one occasion, it is possible that the impact of these risk factors may be even larger than assessed here.

The accuracy of some of the data may be compromised because they were based on self-reports and not objectively verified and they were assessed retrospectively. Therefore, reporting bias is possible and some of the results should be regarded as estimates rather than objective figures. Imprecise assessment and analyses of a large number of parameters may result in spurious associations claiming statistical "independance" for a particular variable. Unfortunately, information about the magnitude of such bias is unavailable in most epidemiologic stud- ies [3]. In addition, given that social status, biologic, behavioural, and psychosocial variables were assessed at the same point in time, it is not possible to disentangle the important relations between these risk factors. A more complete explanation would require an understanding of why people in low social classes are more likely to display the constellation of biologic risk factors, behaviours, and psychosocial characteristics that increase their risk of late complications.

In conclusion, the present population-based study shows that in the district of North-Rhine, Germany a high standard of care has been achieved for adult patients with Type I diabetes especially with respect to the implementation of intensified insulin treatment and education regimens. Improvement of care for patients with late complications is warranted. A substantial social gradient is apparent in the care of Type I diabetic patients, which is not due to insufficient facilities for diagnoses and treatment offered by the present German health care system.

Acknowledgements. This study has been funded by a grant of the Public Health Research Group of North-Rhine-Westfalia (project II-C7). We acknowledge the excellent co-operation with all the participating familiy physicians; G. Paletta, study secretary, J. Brötz, data documentation, D. Hemmann, S. Glück, Dr. T. Heise, Dr. E. Lipka, C. Molina-de-Schneider, Dr. A. Trocha, Dr. E. Wessel, Dr. Ch. Weyer, Dr. R. Windecker, and Dr. S. Geyer, Heinrich-Heine Universität Düsseldorf; Dr. H.-G. Huber and Prof. Dr. J.-D. Hoppe, physicians' chamber of North-Rhine; Boehringer Mannheim (Mannheim, Germany) for providing the mobile examination van; Peter-Klöckner Stiftung (Duisburg, Germany) for generous financial support (grants to Prof. Dr. M. Berger).

Contributors: I. Mühlhauser was the principal investigator and author of this paper, responsible for inception and design, organisation and performance of the study, and data preparation. H. Overmann has contributed to the preparation of the evaluation protocols, organisation of the study and was the principal investigator responsible for data collection. R. Bender, as statistician, was involved in the planning of the study, and was responsible for analysis and interpretation of the data. U. Bott was the principal investigator with respect to the quality of life parameters. V. Jörgens and M. Berger were coprincipal investigators involved in planning and organisation of the study, data interpretation and writing of the manuscript. $\mathrm{Ch}$. Trautner was involved in the planning of the study, data interpretation and preparation of the manuscript. J. Siegrist was the main expert on medical sociology contributing to defining the study hypotheses, providing the evaluation protocols for social parameters and contributing to the analysis and interpretation of the social data.

\section{References}

1. Berger M, Mühlhauser I (1995) Implementation of intensified insulin therapy: a European perspective. Diabet Med 12: 201-208

2. Marmot MG, Shipley MJ, Rose G (1984) Inequalities in death: specific explanations of a general pattern. Lancet i:1003-1006 
3. Lynch JW, Kaplan GA, Cohen RD, Tuomilehto J, Salonen JT (1996) Do cardiovascular risk factors explain the relation between socioeconomic status, risk of all-cause mortality, cardiovascular mortality, and acute myocardial infarction? Am J Epidemiol 144: 934-942

4. Smith GD, Hart C, Blane D, Gillis C, Hawthorne V (1997) Lifetime socioeconomic position and mortality: prospective observational study. Br Med J 314: 547-552

5. Dorman JS, Tajima N, LaPorte RE et al. (1985) The Pittsburgh insulin-dependent diabetes mellitus (IDDM) morbidity and mortality study: case-control analyses of risk factors for mortality. Diabetes Care 8 [Suppl 1]:54-60

6. Matsushima M, Shimizu K, Maruyama M, Nishimura R, LaPorte RE, Tajima N for the Diabetes Epidemiology Research International (DERI) US-Japan Mortality Study Group (1996) Socioeconomic and behavioural risk factors for mortality of individuals with IDDM in Japan: population-based case-control study. Diabetologia 39: 710-716

7. Rossing P, Hougaard P, Borch-Johnsen K, Parving HH (1996) Predictors of mortality in insulin dependent diabetes: 10 year observational follow up study. Br Med J 313: 779-784

8. Hoffmeister H, Hüttner H, Stolzenberg H, Lopez H, Winkler J (1992) Sozialer Status und Gesundheit. Nationaler Gesundheits-Survey 1984-1986. Schriftenreihe des Bundesgesundheitsamtes. MMV Medizin Verlag, München

9. Helmert U, Mielck A, Classen E (1992) Social inequalities in cardiovascular disease risk factors in East and West-Germany. Soc Sci Med 35: 1283-1292

10. Schmucker B (1997) Sozialstatus einer Populations-bezogenen Gruppe von erwachsenen Personen mit Typ 1 Diabetes im Vergleich zur Gesamtbevölkerung von Nordrhein-Westfalen. Magister thesis public health, University Düsseldorf, Düsseldorf

11. Mühlhauser I, Overmann H, Bott U, Jörgens V, Berger M (1996) Erfassung der Versorgungsqualität von erwachsenen Personen mit Typ-I-Diabetes im Ärztekammerbereich Nordrhein. In: Berger M, Trautner C (eds) Die Forderungen von St. Vincent - Stand 1996 in Deutschland, Kirchheim-Verlag, Mainz, pp 97-104

12. Mühlhauser I, Sulzer M, Berger M (1992) Quality assessment of diabetes care according to the recommendations of the St. Vincent Declaration: a population-based study in a rural area of Austria. Diabetologia 36: 429-435

13. Jörgens V, Grüsser M, Bott U, Mühlhauser I, Berger M (1993) Effective and safe translation of intensified insulin therapy to general internal medicine departments. Diabetologia 36: 99-105
14. Mühlhauser I, Bott U, Overmann H et al. (1995) Liberalized diet in patients with type 1 diabetes. J Int Med 237: 591-597

15. Mühlhauser I, Bender R, Bott U et al. (1996) Cigarette smoking and the progression of retinopathy and nephropathy in type 1 diabetes. Diabet Med 13: 536-543

16. Sawicki PT, Heinemann L, Berger M (1989) Comparison of methods for determination of microalbuminuria in diabetic patients. Diabet Med 6: 412-415

17. Liniger C, Albeanu A, Bloise D, Assal JP (1990) The tuning fork revisited. Diabet Med 7: 859-864

18. Kroll P (1993) Augenfachärztlicher Untersuchungsbogen zur Früherkennung diabetischer Augenerkrankungen. Der Augenarzt 27: 19-28

19. Ferris FL, Kassoff A, Bresnick GH, Bailey I (1982) New visual acuity charts for clinical research. Am J Ophthalmol 94: 91-96

20. Bott U, Mühlhauser I, Overmann H, Berger M (1998) Validation of a diabetes-specific quality of life scale for patients with type 1 diabetes. Diabetes Care 21: 757-769

21. Klein R, Klein BEK, Moss SE, Cruickshanks KJ (1996) The medical management of hyperglycemia over a 10-year period in people with diabetes. Diabetes Care 19: 744-750

22. The Diabetes Control and Complications Trial Research Group (1993) The effect of intensive treatment of diabetes on the development and progression of long-term complications in insulin-dependent diabetes mellitus. N Engl J Med 329: 977-986

23. Harris MI, Eastman RC, Siebert C (1994) The DCCT and medical care for diabetes in the U.S. Diabetes Care 17: 761-764

24. Mühlhauser I, Sawicki P, Didjurgeit U, Jörgens V, Berger M (1988) Uncontrolled hypertension in type 1 diabetes: assessment of patients' desires about treatment and improvement of blood pressure control by a structured treatment and teaching programme. Diabet Med 5: 693-698

25. Sawicki PT, Mühlhauser I, Didjurgeit U, Baumgartner A, Bender R, Berger M (1995) Intensified antihypertensive therapy is associated with improved survival in type 1 diabetic patients with nephropathy. J Hypertension 13: 933-938

26. Michaelis D, Jutzi E, Heinke P (1993) 30 jähriger Inzidenzund Prävalenztrend des juvenilen Typ-I-Diabetes in der ostdeutschen Bevölkerung. Diabetes \& Stoffwechsel 2: 245-250

27. Christau B, Akerblom H, Joner G et al (1979) Incidence of insulin-dependent diabetes mellitus (0-29 year at onset) in Denmark. Acta Med Scand 24 [Suppl]:54-60 